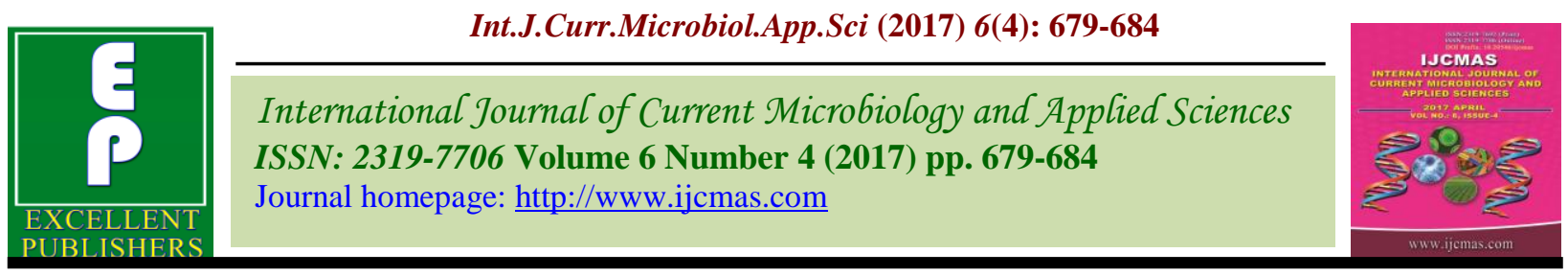

Original Research Article

https://doi.org/10.20546/ijcmas.2017.604.083

\title{
Incidence, Risk Factors and Outcome of Ventilator Associated Pneumonia at SRM Medical College Hospital -A Study under HICC
}

\author{
Sneka*, V. Sangamithra, Shabana Praveen, Manonmoney and Mangaiyarkarasi \\ Department of Microbiology, SRM Medical College \& RI, Chennai, India \\ *Corresponding author
}

\begin{abstract}
A B S T R A C T
Keywords

Pneumonia,

VAP,

Acinetobacter,

ICU,

Mechanical

ventilation.

Article Info

Accepted:

06 March 2017

Available Online:

10 April 2017

Ventilator associated pneumonia is a Hospital acquired pneumonia that develops exclusively in patients undergoing mechanical ventilation. This study was conducted to find the incidence and Risk factors of VAP and the outcome of the patients developing VAP. The study was conducted over a period of 3 months in Intensive Care Units (Pediatric ICU, Surgical ICU, Medical ICU) of SRM Medical College Hospital. A total of 30 patients who were kept on mechanical ventilator were randomly selected. Gram staining and bacterial culture and sensitivity was done. Out of 30 patients, 17 patients developed VAI. The risk factors significantly associated with VAI in our study was found to be the duration of mechanical ventilation, advanced age, associated disease like Diabetes mellitus and the level of consciousness of the patient. The most common organism isolated in our study was Acinetobacter spp. The incidence of early-onset VAP (within $96 \mathrm{~h}$ ) was found to be $27 \%$ while the late-onset VAP ( $>96 \mathrm{~h}$ ) was $73 \%$. Late-onset had poor prognosis in terms of mortality $(66 \%)$ as compared to the early-onset type (20\%). In conclusion, the incidence of Ventilator associated infection was directly proportional to increased duration of mechanical ventilation. Late-onset Ventilator associated infections were multidrug resistant associated with poor prognosis and increased mortality as compared to the early-onset variety.
\end{abstract}

\section{Introduction}

Ventilator-associated pneumonia (VAP) is the most common nosocomial infection diagnosed in the intensive care units (ICUs). VAP is defined as pneumonia that occurs $48 \mathrm{~h}$ or more after endotracheal intubation or tracheostomy, caused by infectious agents not present or incubating at the time of mechanical ventilation (American Thoracic Society, 2005). Ventilator- associated pneumonia (VAP) increases the crude mortality rate by $2-10$ times, and the hospital costs by increasing the length of stay and the need for more expensive antibiotics (Chastre et al., 2002). VAP requires a rapid diagnosis and initiation of appropriate antibiotic treatment, as there is adverse effect of inadequate antibiotic treatment on patients' prognosis and the emergence of multidrugresistant (MDR) pathogens. Inadequate antimicrobial therapy, such as inappropriate antimicrobial coverage, or delayed initiation of antimicrobials has been associated with higher hospital mortality in subjects with hospital acquired pneumonia (Bercault et al., 2001; Vallés et al., 2007).

The clinical diagnosis based on purulent sputum may follow intubation or oropharyngeal secretion leakage around airway, chest X-ray changes suspected of VAP may also be a feature of pulmonary edema, pulmonary infarction, atelectasis or 
acute respiratory distress syndrome. In fact, it was proven that colonization of airway is common and presence of pathogens in tracheal secretions in the absence of clinical findings does not suggest VAI. Patients in the intensive care unit (ICU) are at risk for dying not only from their critical illness but also from secondary processes such as nosocomial infection.

Pneumonia is the second most common nosocomial infection in critically ill patients, affecting $27 \%$ of all critically ill patients (Richards et al., 1999). Eighty-six percent of nosocomial pneumonias are associated with mechanical ventilation and are termed ventilator-associated pneumonia (VAP). Between 250,000 and 300,000 cases per year occur in the United States alone, which is an incidence rate of 5 to 10 cases per 1,000 hospital admissions (McEachern et al., 1998; Melsen et al., 2011). The mortality attributable to VAP has been reported to range between 0 and 50\% (Baker et al., 1996; Craig et al., 1984; Cunnion et al., 1996; Kappstein et al., 1992). Studies have provided different results when determining attributable mortality, in part because of very different populations (less-acute trauma patients, acute respiratory distress syndrome [ARDS] patients, and medical and surgical ICU patients) and in part as a result of variances in appropriate empirical medical therapy during the initial 2 days. Furthermore, the organisms recovered have an impact on outcome, with higher mortality rates seen in VAP caused by Pseudomonas aeruginosa, Acinetobacter spp., and Stenotrophomonas maltophilia (Papazian et al., 1996). Beyond mortality, the economics of VAP include increased ICU lengths of stays (LOS) (from 4 to 13 days), and incremental costs associated with VAP have been estimated at between $\$ 5,000$ and $\$ 20,000$ per diagnosis (Kollef et al., 1995; Boyce et al., 1991; van Nieuwenhoven et al., 2004).
Diagnosing VAP requires a high clinical suspicion combined with bedside examination, radiographic examination, and microbiologic analysis of respiratory secretions. Aggressive surveillance is vital in understanding local factors leading to VAP and the microbiologic milieu of a given unit. Judicious antibiotic usage is essential, as resistant organisms continue to plague intensive care units and critically ill patients. Simple nursing and respiratory therapy interventions for prevention should be adopted. Over the past several decades our understanding of VAP has grown significantly with regard to pathogenesis, risk factors, diagnostic testing, therapies, and prevention by modifying risk factors. This paper will enumerate the incidence, Risk factor and Outcome of Ventilator Associated Pneumonia in Intensive Care Units of a tertiary care hospital.

The main aim of this study to analyse the incidence, risk factor and outcome of ventilator associated pneumonia at SRM Medical College Hospital.

\section{Materials and Methods}

\section{Study type}

This prospective study was planned and carried out in surgical, medical and paediatric intensive care units (ICUs) of SRM Medical College Hospital for 3 months period (October - December 2016). The study protocol was approved by the Scientific and ethical committee of the institution. Informed consent was obtained from the patients before they were included in the study.

\section{Study population}

A total of 30patients who were kept on mechanical ventilation were randomly selected. Cases included in the study were 
patients of both sexes irrespective of their age who were kept on mechanical ventilation. A questionnaire was prepared which included the details of the patient like age, sex, date of admission to the intensive care unit, date of initiating mechanical ventilation and indication for mechanical ventilation. Risk factor if present and final outcome of the patients were also noted.

\section{Laboratory procedures}

Routine laboratory investigations and microbiological battery of investigations like Gram staining and culture of the samples like tracheal aspirate / broncho alveolar lavage were performed on MacConkey agar, Blood agar and Chocolate agar. The organisms isolated were subjected to sensitivity testing with set of antibiotics on Mueller Hinton agar according to CLSI guidelines.

\section{Results and Discussion}

The study included 30 patients with various diagnosis like poisoning, neurological disorders, sepsis etc (Table 1). Out of the 30 patients in the study group, 16 were males and the rest 14 were females. Of the 30 cases kept on Mechanical ventilator $17(56.6 \%)$ patients developed VAP during their stay in ICU.

The incidence of VAP was high in males 11 $(64.7 \%)$ males than females $6(35.3 \%)$.There was male predominance. The mean age group in our study was 34 years.

In patients who developed VAP early onset VAP (before 3 days of initiating mechanical ventilation) was noted in $3(17.6 \%)$ patients and late onset VAP (after 3 days of initiating mechanical ventilation) was noted in 14 (82.3 $\%$ )patients (Table 2). The duration of mechanical ventilation was an important risk factor for the development of ventilator associated infections in our study.
Level of consciousness has a significant impact on the incidence of ventilator associated infections. In this study it was found that the incidence of Ventilator associated infections in stuporus and comatose $(76.47 \%)$ patients was higher than that in conscious and drowsy (23.53\%) patients.

Patients with history of Diabetes mellitus (6 patients), Tuberculosis (1 patient) had VAI in this study. Immunocompromised health status favored the bacterial growth in these patients.

The order of prevalence of organism in this study was found to be $7(41.1 \%)$ isolates of Acinetobacter spp. followed by $4(23.5 \%)$ isolates of Klebsiella pneumoniae, 2(11.7\%) isolates of Pseudomonas aeroginosa, $2(11.7 \%)$ isolates of Escherichia coli and 2 $(11.7 \%)$ isolates of CONS (Figure 1).

Acinetobacter spp was sensitive to amikacin, gentamycin, imipenem and colistin and resistant to cotrimoxazole and ciprofloxacin. Klebsiella spp. is sensitive to Ofloxacin, Imipenem and Amikacin. Almost all the Klebsiella Spp. is resistant to Ceftazidime, ciprofloxacin followed by Ceftriaxone. Similarly in case of E.Coli, it was sensitive to Amikacin, Ofloxacin and Imipenem. Pseudomonas aeroginosa was sensitive to piperacillin/Tazobactem, Imipenem and Doripenem andresistant to amikacin, ceftazidine, Ciprofloxacin and cotrimoxazole. ESBL production was detected in 3 strain of Klebsiella pneumonia and 1 strain of Escherichia coli and MRCONS was detected in one strain of Coagulase Negative Staphylococcus.

The present study comprised 30 patients of various diagnoses like poisoning, neurological disorders, sepsis etc. Out of the total 30patients, 16 were males and the rest 14 were females. Among them 17 patients 
developed VAI during their ICU stay. There was a male predominance $11(64.71 \%)$ than females $6(35.29 \%)$. The mean age group in our study was 34 years. Number of cases of poisoning was predominant in this study.

In the present study patients kept on mechanical ventilation for $>3$ days had a higher incidence of VAI, which was in similar to some other studies also. The incidence of early onset infections within 3 days was found to be $17.6 \% \%$ while the late onset type more than 3 days was $83.4 \%$.The duration of mechanical ventilation was an important risk factor for ventilator associated infections. In the present study morality rate was more among late onset VAI (53\%)

One patient with history of Tuberculosis and Six other patients with the history of Diabetes had Ventilator associated infections. Immuno compromised health status enhanced the bacterial growth in these patients. These bacteria developed multi drug resistance when compared to other persons without any risk factors. So this study proves that the patient with lower level of immune status, acquired infection with bacteria showing multidrug resistance than the other patients on ventilation.

Level of consciousness had a significant impact on the incidence of Ventilator associated infections. It was found in our study that the incidence of Ventilator associated infections in stuporous and comatose patients was higher $(76.47 \%)$ than that in conscious and drowsy (23.53\%) patients. This may be due to the higher chances of aspiration in comatose patients. This is similar to other studies, where the level of consciousness played a role in developing VAI.

The most common cause of VAP in this study was Acinetobacter spp followed by klebsiella pneumoniae followed by Pseudomonas aeroginosa, Escherichia coli and CONS. The overall mortality in the VAI patients was found to be $54 \%$ which was definitely higher than the other ward patients.

Table.1 Sex differences of VAP isolates

\begin{tabular}{|lll|} 
& Male & Female \\
\hline Total(30) & 16 & 14 \\
\hline Positive(17) 56.5\% & $11(68.7 \%)$ & $6(35.3 \%)$ \\
\hline
\end{tabular}

Table.2 Percentage of Early and Late VAP isolates

\begin{tabular}{|lcc|}
\hline Type of VAP & Total & Percentage \\
\hline Early VAP & $3 / 17$ & 17.6 \\
\hline Late VAP & $14 / 17$ & 82.4 \\
\hline
\end{tabular}




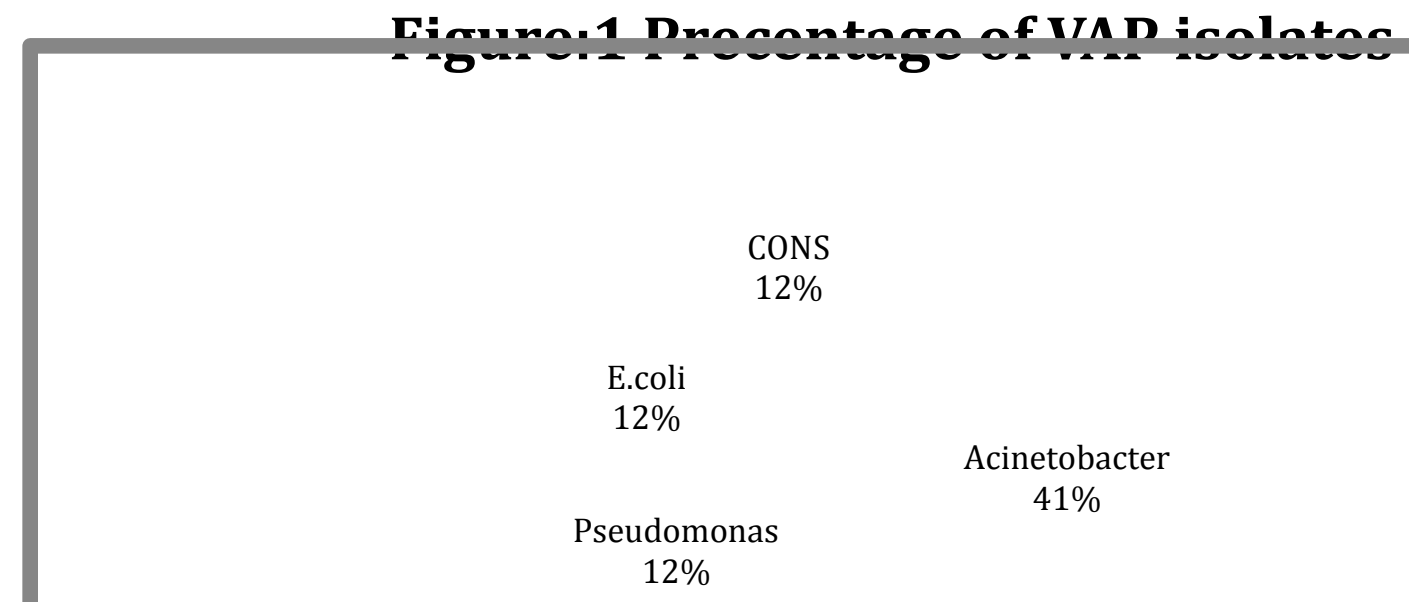

In conclusion Ventilator-associated infection is one of the most common infections acquired by adults and children in ICU's. VAI is a cause of significant mortality, morbidity, increased use of antimicrobial agents and prolonged hospital stay. Thereby, it causes financial and economic burden to the patients. Acinetobacter spp was the most common organism isolated in association with VAI in this study. The incidence of Ventilator associated infection was directly proportional to increased duration of mechanical ventilation. Late-onset Ventilator associated infections were associated with poor prognosis and increased mortality as compared to the early-onset VAP. Thus, it is important to adopt measures in intensive care units to prevent VAI like adhering to hand hygiene, head end elevation and proper or pharyngeal suctioning. To prevent aspiration, proper oral hygiene, in line suctioning and head elevation can be done. Measures to minimize the duration of ventilation would help in decreasing the risk of developing Ventilator-associated infection.

\section{References}

American Thoracic Society. 2005. Infectious Diseases Society of America: Guidelines for the management of adults with hospital-acquired, ventilator-associated, and healthcareassociated pneumonia. Am. J. Respir. Crit. Care Med., 171: 388-416.

Baker, A.M., J.W. Meredith, and E.F. Haponik. 1996. Pneumonia in intubated trauma patients. Microbiology and outcomes. Am. J. Respir. Crit. Care Med., 153: 343349.

Bercault, N., Boulain, T. 2001. Mortality rate attributable to ventilator-associated nosocomial pneumonia in an adult intensive care unit: a prospective casecontrol study. Crit. Care Med., 29: 2303-2309. doi: 10.1097/00003246200112000-00012

Boyce, J.M., G. Potter-Bynoe, L. Dziobek, and S.L. Solomon. 1991. Nosocomial pneumonia in Medicare patients. Hospital costs and reimbursement patterns under the prospective payment system. Arch. Intern. Med., 151: 1109-1114.

Chastre, J., Fagon, J.Y. 2002. Ventilatorassociated pneumonia. Am. J. Respir. Crit. Care Med., 165: 867-903.

Craig, C.P., and S. Connelly. 1984. Effect of intensive care unit nosocomial pneumonia on duration of stay and 
mortality. Am. J. Infect. Control, 12: 233-238.

Cunnion, K.M., D.J. Weber, W.E. Broadhead, L.C. Hanson, C.F. Pieper, and W.A. Rutala. 1996. Risk factors for nosocomial pneumonia: comparing adult critical-care populations. Am. J. Respir. Crit. Care Med., 153: 158162.

Kappstein, I., G. Schulgen, U. Beyer, K. Geiger, M. Schumacher, and F.D. Daschner. 1992. Prolongation of hospital stay and extra costs due to ventilator-associated pneumonia in an intensive care unit. Eur. J. Clin. Microbiol. Infect. Dis., 11: 504-508.

Kollef, M.H., P. Silver, D.M. Murphy, and E. Trovillion. 1995. The effect of lateonset ventilator-associated pneumonia in determining patient mortality. Chest, 108: 1655-1662.

McEachern, R., and G.D. Campbell, Jr. 1998. Hospital-acquired pneumonia: epidemiology, etiology, and treatment. Infect. Dis. Clin. N. Am., 12: 761-779.

Melsen, W.G., Rovers, M.M., Koeman, M., Bonten, M.J. 2011. Estimating the attributable mortality of ventilatorassociated pneumonia from randomized prevention studies. Crit. Care Med., 39: 2736-42.

Papazian, L., F. Bregeon, X. Thirion, R. Gregoire, P. Saux, J.P. Denis, G. Perin, J. Charrel, J.F. Dumon, J.P. Affray, and F. Gouin. 1996. Effect of ventilator-associated pneumonia on mortality and morbidity. Am. J. Respir. Crit. Care Med., 154: 91-97.

Richards, M.J., J.R. Edwards, D.H. Culver, R.P. Gaynes, et al. 1999. Nosocomial infections in medical intensive care units in the United States. Crit. Care Med., 27: 887-89.

Vallés, J., Pobo, A., García-Esquirol, O., Mariscal, D., Real, J., Fernández, R. 2007. Excess ICU mortality attributable to ventilator-associated pneumonia: the role of early vs late onset. Intensive Care Med., 33: 13631368.doi:10.1007/s00134-007-0721-0

van Nieuwenhoven, C.A., E. Buskens, D.C. Bergmans, F.H. van Tiel, G. Ramsay, and M.J. Bonten. 2004. Oral decontamination is cost-saving in the prevention of ventilator-associated pneumonia in intensive care units. Crit. Care Med., 32: 126-130.

\section{How to cite this article:}

Sneka, V. Sangamithra, Shabana Praveen, Manonmoney and Mangaiyarkarasi. 2017. Incidence, Risk factors and Outcome of Ventilator Associated Pneumonia at SRM Medical College Hospital -A study under HICC. Int.J.Curr.Microbiol.App.Sci. 6(4): 679-684. doi: https://doi.org/10.20546/ijcmas.2017.604.083 